\title{
Internet-delivered assessment and manipulation of anxiety-linked attentional bias: Validation of a free-access attentional probe software package
}

\author{
Colin Macleod, Lih Yi Soong, Elizabeth M. Rutherford, and Lynlee W. Campbell \\ University of Western Australia, Crawley, Western Australia, Australia
}

\begin{abstract}
It recently has been established within the laboratory that the attentional probe methodology not only can sensitively assess the threat attentional bias associated with anxiety vulnerability, but also can be configured to yield training tasks capable of modifying emotional vulnerability by manipulating such attentional selectivity. In order to appraise and exploit the potential practical applications of such procedures, it is desirable that clinical investigators without specialist equipment should become able to deliver such tasks within field settings. The present research program involved development of a fully customizable attentional probe software package, that delivers assessment and training versions of the probe task across the Internet. Two experimental studies served to validate the assessment and training efficacy of resulting probe task variants, completed remotely by GAD sufferers and nonclinical populations using their own computers. We advise fellow researchers how to freely download this software package for use within their own investigations.
\end{abstract}

Research over the last two decades has shown that clinical anxiety is associated with a pervasive tendency to selectively orient attention toward threatening information (cf. MacLeod \& Rutherford, 2004; Mogg \& Bradley, 2005).The most rigorous methodology for assessing this bias is provided by the attentional probe task, which typically exposes participants to pairs of differentially valenced stimulus words, then requires them to discriminate small probe stimuli presented to the locations where either word appeared (MacLeod, Mathews, \& Tata, 1986). The common finding that clinical anxiety patients, and other high trait anxious participants, display speeded discrimination of probes presented in the vicinity of threat words, has served to confirm that such individuals selectively attend to the locus of threatening stimuli (Bradley, Mogg, White, Groom, \& deBono, 1999; Pineles \& Mineka, 2005; Yiend \& Mathews, 2001). Variants of this attentional probe task have now also proven capable of directly manipulating attentional response to threat, by presenting probes consistently in the location of threat words or consistently in the opposite location, and this has been found to systematically modify emotional reactivity in mid-range trait anxious individuals (MacLeod, Rutherford, Campbell, Ebsworthy, \& Holker, 2002). Furthermore, preliminary findings suggest that extended exposure to such attentional probe training procedures, configured to induce attentional avoidance of threat, can reduce anxiety symptomatology in both high trait anxious normals and in clinically anxious patients (Amir, Selvina, Elias, \& Rousseau, 2002; Vasey, Hazen, \& Schmidt, 2002). Such findings have excited interest in the potential clinical utility of this technique, either alone or as an adjunct to conventional clinical treatments for anxiety.

However, opportunities to address this important issue are compromised by the fact that many clinical researchers working in field settings lack ready access to the programming support needed to develop appropriate variants of this attentional probe procedure. Furthermore, it can be difficult to conveniently deliver the task to participants outside the laboratory setting, which creates particular problems for research designs that require repeated short attentional training sessions, perhaps carried out several times each week across an extended period of time. Consequently, given the growth in the successful use of Internet-delivered psychological tools (e.g., Devineni \& Blanchard, 2005; Luce et al., 2005), including assessments of, and treatments for anxiety disorders (cf. Andersson, Bergstrom, Carlbring, \& Lindefors, 2005), we set out to construct and evaluate a downloadable software package that will permit the attentional probe task to be customized according to researchers' aims and interests and delivered online across the Internet.

Despite their obvious advantages, there are several factors that might compromise the sensitivity of Internetdelivered psychological tools (see Birnbaum, 2004). These include the inability to ensure a standard test environment and computer set up; the technical challenges associated with achieving sensitive measurement, as in the measurement of reaction times; and the lack of opportunity to tightly guide and constrain participant behavior through direct observation and appropriate clarification of

C.MacLeod, colin@psy.uwa.edu.au 
instructions. Therefore, following its development, it was essential to empirically validate our Internet-delivered attentional probe task, by verifying its capacity to replicate those established effects that have been obtained using conventional variants of the task within the laboratory setting. Specifically, we wished to establish that this Internet-delivered attentional probe task can be employed effectively as an assessment tool, sensitive to the threat attentional bias known to discriminate Generalized Anxiety Disorder patients from nonanxious controls within the laboratory setting. We also wanted to establish that this Internet-delivered attentional probe task can be employed to modify attentional response to threat, when the same training contingency is introduced as has proven effective within the experimental laboratory.

In summary, the goals of this present research program were to develop a fully customizable software package capable of delivering assessment and training variants of the attentional probe task across the Internet; to confirm in Study 1 that an appropriately configured version of this task can sensitively detect online the threat attentional bias that characterizes GAD sufferers; to confirm in Study 2 that an appropriately configured variant of this task can serve as a tool for remotely modifying attentional response to threat; and to make this validated software package freely available to other investigators wishing to download it from the Internet.

\section{The Online Attentional Probe Software Package}

The software package that we produced is written in JAVA, to facilitate Internet delivery and to ensure platform independence at the point of access. It is designed to be installed on either a UNIX server or a Windows NT server, thus making it compatible with the great majority of large and small scale computer networks. Once installed and configured, the resulting attentional probe task can be remotely accessed and completed on any personal computer connected to the Internet and set to $800 \times 600$ screen resolution, with the resulting data being automatically e-mailed to whatever address is set by the experimenter.

The software package provides for the experimenter to present any number of questions prior to commencement of the probe task, making it possible to deliver online versions of appropriate assessment instruments. Each trial of the probe task begins with the appearance of three adjacent crosses in the center of the screen, which serve as a fixation point. These disappear $500 \mathrm{msec}$ later, when two words are presented, separated on the vertical axis of the computer screen by a visual angle of between $2^{\circ}$ and $3^{\circ}$ (assuming monitor size to be between 14 and 19 in., and seating distance to be approximately 2 feet). These words then disappear, and a probe stimulus appears in a random letter position within the space previously occupied by either of the two preceding words. This probe can be either the symbol "<" or " $>$," and participants are required to indicate its identity by pressing either the " $\leftarrow$ " or the " $\rightarrow$ "arrow key, respectively. The software records the response latency as the interval, in msec, elapsing between the presentation of the probe and detection of the buttonpress response.
Virtually all other parameters of the attentional probe task can be configured by the experimenter. The software permits the researcher to determine the number of trials to be presented, to enter the stimulus set(s) to be employed, to specify which word position the probe is presented to on each trial, and to set key temporal variables such as word exposure duration and intertrial interval. Consequently, the software package permits researchers to implement attentional probe task variants that either correspond closely to those employed in previous assessment and training studies carried out within the laboratory, or that are entirely novel in their configurations.

\section{STUDY 1 \\ Validation of Capacity to Assess Attentional Bias Online}

The aim of this first study was to test the capability of an Internet-delivered attentional probe task, configured using this software package, to detect the increased vigilance for negative stimuli known to distinguish GAD participants from nonanxious control participants. Such sensitivity will be confirmed by the finding that a threat bias index, calculated to reflect degree of speeding to discriminate probes in the vicinity of threat words rather than nonthreat words, is disproportionately great for participants who report GAD symptomatology.

\section{Method}

Participants. Participants were recruited through advertisements for experimental volunteers, posted on Internet bulletin boards. Some of these bulletin boards were general discussion forums and some were specifically designed for people with anxiety problems. Because it was critical to establish that the clinical sample met diagnostic criteria for GAD, all volunteers completed an online symptom checklist (described below) to establish whether they met DSM IV criteria for GAD. Those who did so were assigned to the GAD group, while only those who reported no current or past history of anxiety symptomatology, and no prior treatment for any form of anxiety disorder, were assigned to the control group. Participants were included only if they indicated that English was their first language, and were excluded if they performed below $90 \%$ accuracy on the probe discrimination task. This resulted in a final sample that comprised 59 participants, 24 of whom met diagnostic criteria for GAD and 35 of whom fulfilled our requirements for nonanxious control participants.

Stimulus words. Stimuli were similar to the 96 word pairs used in the attentional probe task carried out by MacLeod et al. (2002). Each pair consisted of one threat word and one nonthreat word, which both were matched in word length and frequency (Kučera \& Francis, 1967).

Online questionnaires. A custom-designed online version of the 42-item Depression Anxiety Stress Scale (DASS; Lovibond \& Lovibond, 1993, 1995) was employed. This scale has good reliability and validity; effectively distinguishes between depression and anxiety (Antony, Bieling, Cox, Enns, \& Swinson, 1998; Brown, Chorpita, \& Barlow, 1998; Crawford \& Henry, 2003), and is particularly appropriate for Internet use because it is a free-access questionnaire. The DASS required respondents to indicate the applicability of each statement "over the past week," using a 4-point scale. All three subscales were employed, DASS-A (anxiety), DASS-D (depression) and DASS-S (stress). A custom-designed electronic GAD diagnostic questionnaire was derived from the DSM-IV diagnostic criteria, with the controlling software operating as a decision tree, presenting subsequent questions on the basis of answers to preceding questions. 
Online attentional probe task configuration. The online attentional probe task was configured to deliver 96 trials, each exposing a stimulus word pair for $500 \mathrm{msec}$, and with trials separated by a $1,000-\mathrm{msec}$ intertrial interval. Across these trials threat word position (upper vs. lower screen position), probe position (upper vs. lower screen position) and probe type ("<" vs. ">”) were balanced such that after every eight participants, each word pair had been presented once under each unique experimental condition. Order of trial presentation was randomized for each participant.

Procedure. Participants accessing the Web site were directed to a page containing information about the experiment and a consent form. If they agreed to participate they were instructed to set their computer monitors to $800 \times 600$ resolution, and to complete the experiment alone in a quiet area, allowing $20 \mathrm{~min}$ without pausing for the completion of all aspects of the experiment. Participants then were given the online DASS questionnaires followed by the GAD symptom questionnaire, before being provided with directions concerning the probe task. They were presented with a frame by frame demonstration of a trial, and instructed to indicate whether a " $<$ " or " $>$ " probe was presented by pressing either the " $\leftarrow$ " or the " $\rightarrow$ " arrow key, respectively, responding as quickly but as accurately as possible. They were told to place their fingers on the two response keys and to press either key to begin. Following completion of the task, participants were thanked for their participation, and provided with debriefing information.

\section{Results}

Preliminary analysis of the data, which were e-mailed automatically to the researcher, revealed that mean probe discrimination accuracy was $97.1 \%$. The groups differed as expected on DASS-A, but also differed on DASS-S and DASS-D (see Table 1).

For the attentional probe task, the dependent measure was the response latency timed from the appearance of each probe until detection of the associated response. A threat bias index (TBI) was computed for each participant, by subtracting median response time to probes presented in the vicinity of threat words from median response time to probes presented in the vicinity of nonthreat words, using a similar procedure adopted in previous research (cf. MacLeod \& Mathews, 1988). A positive TBI indicates attentional vigilance for threat, and a negative TBI indicates attentional avoidance of threat. A one-way ANOVA was conducted on these TBI scores, with Anxiety Group (GAD vs. Controls) as the between groups factor. There was a significant main effect of Anxiety Group $[F(1,57)=10.72, p<.01]$, reflecting the fact that, as expected, the GAD participants had a positive TBI score $(M=+23.77, S D=39.45)$ consistent with attentional vigilance for threat, that differed from the control participants' negative TBI score $(M=-6.14, S D=30.65)$, itself consistent with attentional avoidance of threat.

To determine the relative contribution of self-reported anxiety, depression, and stress to the pattern of observed effects, correlational analyses first were carried out. The

Table 1

Mean Scores on DASS in Study 1

\begin{tabular}{lccccccc}
\hline & \multicolumn{2}{c}{$\begin{array}{c}\text { Clinically } \\
\text { Anxious }\end{array}$} & & \multicolumn{2}{c}{$\begin{array}{c}\text { Nonanxious } \\
\text { Controls }\end{array}$} \\
\cline { 2 - 3 } & $M$ & $S D$ & & $M$ & $S D$ & $F(1,57)=$ & $p<$ \\
\hline DASS-A & 19.33 & 10.14 & & 3.63 & 4.88 & 63.10 & .001 \\
DASS-S & 28.08 & 11.06 & & 7.97 & 7.36 & 70.52 & .001 \\
DASS-D & 23.04 & 12.60 & & 6.77 & 9.83 & 30.97 & .001 \\
\hline
\end{tabular}

Table 2

Correlations Between DASS-A, DASS-D, DASS-S, and TBI in Study 1

\begin{tabular}{lcccc}
\hline & DASS-A & DASS-D & DASS-S & TBI \\
\hline DASS-A & - & $.75^{* *}$ & $.87^{* *}$ & $.36^{* *}$ \\
DASS-D & & - & $.87^{* *}$ & $.32^{*}$ \\
DASS-S & & & - & $.34^{* *}$ \\
TBI & & & & -
\end{tabular}

${ }^{*} p<.05$ (two-tailed). ${ }^{* *} p<.01$ (two-tailed).

results, shown in Table 2, revealed that TBI scores were positively correlated with scores on the DASS-A, the DASS-S, and the DASS-D. However only anxiety level, as measured by DASS-A scores, emerged from a stepwise regression analysis as a significant independent predictor of TBI $[t(57)=2.91, p<.01]$.

\section{Discussion}

The main aim of Study 1 was to confirm that this Internet-delivered attentional probe task is sensitive to the anxiety-linked attentional bias that previously has been found in GAD participants using laboratory based versions of the probe procedure (e.g., MacLeod et al., 1986; Bradley et al., 1999). The online task did indeed reveal that the GAD participants showed attentional vigilance for threat, while nonanxious controls showed attentional avoidance of threat. The role of self-reported anxiety in mediating this effect, as opposed to depression or stress, was supported by the results of regression analysis. Thus, the study has established the sensitivity of our Internet-delivered probe assessment task to reveal the pattern of selective attention known to discriminate GAD and control participants.

\section{STUDY 2 Validation of Capacity to Manipulate Attentional Bias Online}

Having established that our online probe task can effectively assess threat attentional bias, the aim of this second study was to confirm the capacity of a differently configured version of this Internet-delivered task to modify attentional response to threat. The reconfigured task variant used in the current study to achieve this training goal was similar to that employed by MacLeod et al. (2002) within the laboratory setting. This involved building a contingency into the standard attentional probe assessment task, designed to encourage participants to selectively orient attention toward or away from emotionally threatening stimuli. One group of participants was exposed to a contingency designed to encourage development of an attentional bias toward threat, which involved the consistent presentation of the probe on each trial to the spatial location occupied by the threat member of the preceding word pair. For a second group of participants this contingency was reversed to yield a training contingency designed to encourage development of an attentional bias away from threat, by having the probe consistently appear in the spatial location occupied by the nonthreat member of the preceding word pair. Following an extended period 
of such training trials, attentional assessment trials were given, which always employed novel stimulus words. Within these assessment trials the contingency between the word valence and the probe location was removed, so that probes appeared equally often in the locations of either emotional class of stimulus words for both groups of participants. These assessment trials served to reveal the efficacy of the attentional training procedure. Specifically, if such training was effective then on these assessment trials the group exposed to the contingency designed to train attentional avoidance of threat should display a relative slowing to discriminate probes in the vicinity of threat rather than neutral words, compared to the group exposed to the reverse attentional training contingency.

As in MacLeod et al.'s (2002) laboratory based training study, the current study administered the attentional training task to a sample of first year psychology students. However, in contrast to the original study, these participants were recruited across the Internet, and completed the training procedure remotely using our online attentional probe task. Confirmation of the predicted effects will indicate that this Internet-delivered attentional training procedure can achieve, via remote delivery, the manipulation of attentional response to threat previously observed only within the experimental laboratory setting.

\section{Method}

Participants. Participants were first year psychology students recruited electronically from universities in English-speaking countries. As in Study 1, any volunteer who displayed a level of probe discrimination accuracy below $90 \%$ was excluded, and the final sample comprised 62 participants. Participants were randomly assigned, without their knowledge, to either of the two training conditions, resulting in 36 participants being allocated to the Avoid Threat condition, and 26 being allocated to the Attend Threat training condition.

Stimulus words. Stimuli were the same 96 word pairs employed in Study 1. These word pairs were divided into four subsets which were randomly rotated across participants, to create a 48 -word pair training set and a 48-word pair assessment set for each participant.

Online attentional probe task configuration. The structure of each trial matched that described for Study 1. However, the present task incorporated both training and assessment trials. Therefore, it was substantially longer than the task used in Study 1, but included only half the number of assessment trials. Specifically, 288 attentional training trials were delivered first, in six blocks of 48 trials, with all items in the training word set being presented once within each block. During these training trials, for one group of participants (Avoid Threat Training Group) the probe always appeared in the vicinity of the nonthreat word member, while for the second group (Attend Threat Training Group) the probe always appeared in the vicinity of the threat word member. Participants then were given 48 attentional assessment trials, which followed the training trials seamlessly, but with the contingency between probe location and word valence now removed, so that the probes appeared equally often in the vicinities of the threat and nonthreat words. Stimulus presentation order within each of the six training blocks and within the assessment block was randomized for each participant.

Procedure. Instructions were equivalent to those delivered in Study 1, and participants completed the 336 trials (288 training and 48 assessment) without a break.

\section{Results}

Preliminary analyses of the data, which again were e-mailed automatically to the researcher, revealed that the mean probe discrimination accuracy score was $97.7 \%$. The dependent measure of central interest was the response latency to probes presented on attentional assessment trials. As before, a threat bias index (TBI) was computed to express the degree to which median response times for probes presented in the vicinity of threat words were speeded relative to those for probes presented in the vicinity of nonthreat words. Once more, therefore, a positive TBI indicated attentional vigilance for threat, and a negative TBI indicated attentional avoidance of threat. A oneway ANOVA was conducted on these index scores, with Training Group (Avoid Threat vs. Attend Threat) as the between groups factor. There was a significant main effect of Training Group $[F(1,60)=5.06, p<.05]$, reflecting the fact that, as predicted, the Avoid Threat participants obtained a negative TBI score $(M=-34.46, S D=101.65)$ consistent with the attentional avoidance of threat, while the Attend Threat participants instead obtained a positive TBI score $(M=+13.10, S D=41.75)$, consistent with attentional vigilance for such negative stimuli.

\section{Discussion}

The aim of this second study was to confirm that attentional training, delivered online using an appropriate configuration of our attentional probe software package, can remotely manipulate patterns of attentional response to threat. Participants given training designed to encourage the development of an attentional bias away from threat did indeed show, on novel stimuli, a pattern of probe detection latencies consistent with the acquisition of such attentional avoidance, that differed as predicted from the pattern displayed by participants given the reverse direction of attentional training. These findings replicate the results obtained by MacLeod et al. (2002), using the original laboratory-based version of this attentional training procedure.

\section{GENERAL DISCUSSION}

The purpose of this research program was to develop a fully customisable software package capable of delivering online versions of the attentional probe task across the Internet, that can serve both to assess and to modify attentional responses to threat stimuli. Such a software package now has been produced, and the results of our two studies confirm that Internet-delivered task variants yielded by this package are indeed capable of fulfilling both goals. In Study 1, GAD participants who completed an assessment version of the attentional probe task remotely, across the Internet, were found to display speeded discrimination latencies for probes in the vicinity of threat, consistent with the orientation of attention toward such stimuli. This pattern of attentional avoidance differed reliably from the pattern shown by nonanxious control participants, who instead were disproportionately slow to discriminate probes in the vicinity of threat stimuli, consistent with attentional avoidance of such information. In Study 2, volunteers exposed remotely to a training version of this Internet delivered attentional probe task, designed to induce attentional avoidance of threat, were indeed found to display slowing 
to discriminate probes in the vicinity of threat, consistent with the acquisition of such a bias. This differed reliably from the pattern shown by those participants exposed to the reverse training contingency, who instead displayed speeding to discriminate such probes, consistent with the acquisition of attentional vigilance for threat.

Setting aside the method of task delivery, neither of these findings can be considered new. As already has been noted, the capacity of laboratory-based versions of the probe task to reveal attentional vigilance for threat in anxious populations is well established (cf. Mathews \& MacLeod, 2005), and more recently it also has been demonstrated that training variants of such laboratory based procedures can serve to modify attentional responses to threat (cf. Mathews, 2004). However, this is the first time that such effects have been obtained using a procedure that permits the remote delivery of these attentional probe tasks, and indeed these present findings appear particularly robust, despite this Internet delivery format. For example, the statistically reliable difference in attentional selectivity between the GAD patients and the controls was observed, in the present task variant, using only 96 assessment trials, while the training effect was achieved after only 288 training trials, and was detected using only 48 assessment trials. Such sensitivity compares well against laboratory-based versions of the attentional probe task, where twice as many trials commonly have been employed to yield similar effects.

In keeping with the views of other researchers who have adapted quite different types of psychological procedures for use on the Internet (e.g., Devineni \& Blanchard, 2005; Luce et al., 2005), we believe that such a form of delivery affords significant practical advantages. The dividends are likely to be especially great in the case of the attentional probe methodology, given the new avenues of research into the therapeutic impact of attentional retraining variants of this procedure that recently have been opened by promising laboratory based studies (Amir et al., 2002; Mathews \& MacLeod, 2002; Vasey et al., 2002). It seems reasonable to anticipate that research progress will be enhanced by rendering such attentional modification procedures more readily accessible to geographically dispersed clients within their home environments.

To make possible such research progress, we have produced a comprehensive user guide for this fully customizable attentional probe software package, and have made the software and guide available at the following address: www.psy.uwa.edu.au/cogemo/AttProbe1.html. Researchers can freely download this package, install it on their own servers, and configure it to deliver any combination of questionnaires, training trials, and assessment trials. They can employ their own choice of stimulus materials, and can specify whatever e-mail address they wish the resulting data to be transmitted to when the task is accessed and completed across the Internet. We very much hope that this package will enable a wider range of clinical researchers to investigate the role of threat attentional bias in mediating and modifying anxiety symptoms across a wider range of clinical patients. We also expect that the resource may facilitate many forms of constructive collaboration, particularly those involving productive associations between researchers and practitioners, which hold such promise of enabling both theoretical and applied advances to be made within this burgeoning area of investigation.

\section{AUTHOR NOTE}

This research was partly supported by an Australian Research Council grant to C.M. We thank Andrew Mathews for his thoughtful feedback on an earlier draft of this article. Correspondence concerning this article should be addressed to C. MacLeod, School of Psychology, University of Western Australia, 35 Stirling Highway, Crawley, Western Australia, 6009 Australia (e-mail: colin@psy.uwa.edu.au).

\section{REFERENCES}

Amir, N., Selvina, A., Elias, J., \& Rousseau, G. (2002). Manipulation of information processing bias in anxiety: Malleability of attention and interpretation biases. Presented at the 36th Annual Convention of the Association for Advancement of Behavior Therapy, Reno, NV.

Andersson, G., Bergstrom, J., Carlbring, P., \& Lindefors, N. (2005). The use of the internet in the treatment of anxiety disorders. Current Opinion in Psychiatry, 18, 73-77.

Antony, M. M., Bieling, P. J., Cox, B. J., Enns, M. W., \& Swinson, R. P. (1998). Psychometric Properties of the 42-Item and 21-Item Versions of the Depression Anxiety Stress Scales in Clinical Groups and a Community Sample. Psychological Assessment, 10, 176-181.

Birnbaum, M. H. (2004). Human research and data collection via the internet. Annual Review of Psychology, 55, 803-832.

Bradley, B. P., Mogg, K., White, J., Groom, C., \& deBono, J. (1999). Attentional bias for emotional faces in generalised anxiety disorder. British Journal of Clinical Psychology, 38, 267-278.

Brown, T. A., Chorpita, B. F., \& Barlow, D. H. (1998). Structural relationships among dimensions of the DSM-IV anxiety and mood disorders and dimensions of negative affect, positive affect, and autonomic arousal. Journal of Abnormal Psychology, 107, 179-192.

Crawford, J. R., \& Henry, J. D. (2003). The Depression Anxiety Stress Scales: Normative data and latent structure in a large nonclinical sample. British Journal of Clinical Psychology, 42, 111-131.

Devineni, T., \& Blanchard, E. B. (2005). A randomized controlled trial of an Internet-based treatment for chronic headache. Behaviour Research \& Therapy, 43, 277-292.

KuČERA, H., \& Francis, W. N. (1967). Computational analysis of present-day American English. Providence, RI: Brown University Press.

Lovibond, P. F., \& LoviBond, S. H. (1993). Manual for the Depression Anxiety Stress Scales (DASS). Psychology Foundation Monograph.

Lovibond, P. F., \& Lovibond, S. H. (1995). The structure of negative emotional states: Comparison of the Depression Anxiety Stress Scales (DASS) with the Beck Depression and Anxiety Inventories. Behaviour Research \& Therapy, 33, 335-343.

Luce, K. H., Osborne, M. I., Winzelberg, A. J., Das, S., Abascal, LIANA, B., ET AL. (2005). Application of an algorithm-driven protocol to simultaneously provide universal and targeted prevention programs. International Journal of Easting Disorders, 37, 220-226.

MacLeod, C., \& Mathews, A. (1988). Anxiety and allocation of attention to threat. Quarterly Journal of Experimental Psychology, 40A, 653-670.

MacLeod, C., Mathews, A., \& Tata, P. (1986). Attentional bias in emotional disorders. Journal of Abnormal Psychology, 95, 15-20.

MacLeod, C., \& Rutherford, E. M. (2004). Information-processing approaches: Assessing the selective functioning of attention, interpretation, and retrieval. In R. G. Heimberg, C. L. Turk, \& D. S. Mennin (Eds.), Generalized anxiety disorder: Advances in research and practice. New York: Guilford.

MacLeod, C., Rutherford, E. M., Campbell, L., Ebsworthy, G., \& HoLKer, L. (2002). Selective attention and emotional vulnerability: Assessing the causal basis of their association through the experimental manipulation of attentional bias. Journal of Abnormal Psychology, 111, 107-123.

Mathews, A. (2004). On the malleability of emotional encoding. Behaviour Research \& Therapy, 42, 1019-1036.

Mathews, A., \& MacLeod, C. (2002). Induced processing biases have causal effects on anxiety. Cognition \& Emotion, 16, 331-354. 
Mathews, A., \& MacLeod, C. (2005). Cognitive approaches to the emotional disorders. Annual Review of Clinical Psychology, 1, 167-195.

Mogg, K., \& Bradley, B. P. (2005). Attentional bias in generalized anxiety disorder versus depressive disorder. Cognitive Therapy $\& R e$ search, 29, 29-45.

Pineles, S. L., \& Mineka, S. (2005). Attentional biases to internal and external sources of potential threat in social anxiety. Journal of Abnormal Psychology, 114, 314-318.

Vasey, M. W., Hazen, R., \& Schmidt, N. B. (2002). Attentional re- training for chronic worry and GAD. Presented at the 36th Annual Convention of the Association for Advancement of Behavior Therapy, Reno, NV.

YiEND, J., \& Mathews, A. (2001). Anxiety and attention to threatening pictures. Quarterly Journal of Experimental Psychology, 54A, 665-681.

(Manuscript received August 11, 2005; revision accepted for publication June 9, 2006.) 